\title{
Measurement and prediction of voice support and room gain in school classrooms
}

\author{
David Pelegrín-García ${ }^{a)}$ and Jonas Brunskog \\ Acoustic Technology, Department of Electrical Engineering, Technical University of Denmark, \\ Kongens Lyngby DK-2800, Denmark \\ Viveka Lyberg-Åhlander and Anders Löfqvist \\ Department of Logopedics, Phoniatrics and Audiology, Lund University, Lund, Sweden
}

(Received 9 May 2011; revised 18 October 2011; accepted 8 November 2011)

\begin{abstract}
Objective acoustic parameters have been measured in 30 school classrooms. These parameters include usual descriptors of the acoustic quality from the listeners' standpoint, such as reverberation time, speech transmission index, and background noise level, and two descriptors of the acoustic properties for a speaker: Voice support and room gain. This paper describes the measurement method for these two parameters and presents a prediction model for voice support and room gain derived from the diffuse field theory. The voice support for medium-sized classrooms with volumes between 100 and $250 \mathrm{~m}^{3}$ and good acoustical quality lies in the range between -14 and $-9 \mathrm{~dB}$, whereas the room gain is in the range between 0.2 and $0.5 \mathrm{~dB}$. The prediction model for voice support describes the measurements in the classrooms with a coefficient of determination of 0.84 and a standard deviation of $1.2 \mathrm{~dB}$. (C) 2012 Acoustical Society of America. [DOI: 10.1121/1.3665987]
\end{abstract}

PACS number(s): 43.55.Gx, 43.55.Fw [NX]

Pages: 194-204

\section{INTRODUCTION}

Learning spaces or classrooms are environments where students spend the early part of their lives listening to their teachers in order to learn. Students also need to communicate efficiently with their classmates and their teachers. The success in communication is essential to help students to develop their full potential. Thus, most of the past research in classroom acoustics has been focused on the acoustic conditions for students. It has been reported that classroom noise has a negative impact on children's speech perception and performance $^{1-3}$ and that reverberation can enhance or degrade speech intelligibility depending on the delay of sound reflections. $^{4,5}$ In addition, the combination of noise and reverberation has been the subject of a number of studies. ${ }^{6-9}$ Different quantities are used to predict speech intelligibility: Signal-to-noise ratios, useful-to-detrimental ratios, and speech transmission index. ${ }^{10,11}$

At the same time, school classrooms are the working place of teachers, who represent an important percentage of the working population. Teachers suffer from voice disorders in a higher proportion than in the rest of the population (around $13 \%$ in Sweden ${ }^{12}$ and a similar proportion in the United States ${ }^{13}$ ), which is most likely due to the high vocal loading that teachers experience at work. Noise and bad classroom acoustics are often reported risk factors for voice disorders. ${ }^{14}$ Talking in the presence of high noise levels results in the use of higher voice power levels than required to talk in softer noise conditions (this is known as the Lombard effect ${ }^{15}$ ), and it is estimated that for each decibel of noise, a speaker raises his voice power level between 0.3 and

\footnotetext{
a) Author to whom correspondence should be addressed. Electronic mail: david.pelegrin@gmx.com
}

$0.6 \mathrm{~dB} .{ }^{16}$ In the presence of low background noise, speakers modify their voice power in different acoustic environments. ${ }^{17,18}$ Other studies have also reported an effect of classroom acoustics on teachers' voices, ${ }^{19,20}$ and there is a concern that excessive absorption may overdamp the voice of the speaker. ${ }^{21}$ Therefore, the classroom acoustics conditions for speakers should also be taken into account during the design of schools.

The acoustic conditions for a speaker are related to the way a speaker perceives his own voice. The voice of a speaker reaches his/her ears by the conduction of vibrations through the body from the larynx to the cochlea and by the sound propagation from the mouth to the ears. The sound propagation of one's own voice can be direct from the mouth to the ears (which is equal in all environments under the same atmospheric conditions) or indirect through reflections at the room boundaries (which depends on the environment). Two magnitudes are proposed by Brunskog et al. ${ }^{17}$ to characterize the acoustic conditions for a speaker: The room gain and the voice support. The room gain is defined as the gain applied by the room boundaries to the voice of the speaker at his/her own ears (considering the airborne direct component as the "normal" voice level) and has been used in several studies. ${ }^{18,22-25}$ The voice support is defined as the level difference between the reflections of one's own voice reaching the ears and the voice propagating directly from the mouth to the ears. It is an alternative way of expressing the room gain, which has a larger dynamic range. Brunskog et al. measured values of room gain between 0 and $0.5 \mathrm{~dB}$ and values of voice support between -15 and $-10 \mathrm{~dB}$ in everyday rooms. The two magnitudes, and in particular the voice support, are conceptually equivalent to Gade's objective support ${ }^{26,27}$ used in the assessment of the acoustic conditions for musicians in concert halls. The room gain and the voice 
support are of interest because they are negatively correlated with the voice power levels used by speakers in different rooms. ${ }^{17,18,22}$ The two measures, room gain and voice support, are further explained in Sec. II.

The goal of this paper is twofold. On the one hand, it aims at developing a prediction model for voice support and room gain, which is based on the diffuse-field theory, on the effects of the head-on external sound fields, and on the sound propagation from the mouth to the ears. On the other hand, it aims at providing a reference set of values for voice support and room gain through measurements in school classrooms, using these measurements to validate the predictions of the model in the same rooms. In addition, physical dimensions and other acoustical parameters of the room (background noise level, reverberation time, and speech transmission index) are also measured to compare the acoustic quality of the classrooms with that of classrooms in other surveys ${ }^{7,28-30}$ and acoustic standards and recommendations for classrooms. ${ }^{31-34}$

\section{ROOM GAIN AND VOICE SUPPORT}

The importance of the indirect component of one's own voice (also referred to as reflected sound), which is produced after the reflections of one's own voice at the room boundaries, is judged with two alternative measures introduced by Brunskog et al.: ${ }^{17}$ The room gain and the voice support. It is assumed that the airborne-direct sound reaching the ears has energy $E_{D}$ and that the reflected sound has energy $E_{R}$. The total airborne sound energy from one's own voice is assumed to be approximately $E_{D}+E_{R}$. The energy levels for the airborne-direct sound $L_{D}$, the reflected sound $L_{R}$, and the total airborne sound $L_{E}$ are

$$
\begin{aligned}
& L_{D}=10 \log \frac{E_{D}}{E_{0}}(\mathrm{~dB}), \\
& L_{R}=10 \log \frac{E_{R}}{E_{0}}(\mathrm{~dB}), \\
& L_{E}=10 \log \frac{E_{D}+E_{R}}{E_{0}}(\mathrm{~dB}),
\end{aligned}
$$

where $E_{0}$ is an arbitrary energy reference.

\section{A. Definition of room gain and voice support}

The room gain $G_{\mathrm{RG}}$ was the first measure introduced by Brunskog et al. ${ }^{17}$ and is defined as the degree of amplification offered by the room to the voice of a speaker at his/her ears, considering only the airborne paths (i.e., not considering the body conducted component). Alternatively, it is also defined as the difference between the energy level of the total airborne sound propagating from the mouth to the ears and the energy level of the direct sound,

$$
G_{\mathrm{RG}}=L_{E}-L_{D}(\mathrm{~dB})
$$

The voice support $\mathrm{ST}_{V}$ is a measure of the strength of the reflected sound relative to the direct sound from one's own voice. It is defined as the difference between the energy level of the reflected sound and the energy level of the airborne direct sound,

$$
\mathrm{ST}_{V}=L_{R}-L_{D}(\mathrm{~dB})
$$

The relationship between the two measures is

$$
G_{\mathrm{RG}} \approx 10 \log \left(10^{\mathrm{ST}_{V} / 10}+1\right)(\mathrm{dB})
$$

assuming that the total airborne sound energy is the sum of the energies of the direct sound and the reflected sound, which is only an approximation.

The nomenclature $\mathrm{ST}_{V}$ used for the voice support is defined after the work of Gade ${ }^{26,27}$ on the acoustics of stages in concert halls, where the measure objective support $\mathrm{ST}_{\text {early }}$ is used to assess the acoustical quality from the performers' point of view.

\section{B. Measurement of room gain and voice support}

Brunskog et al. ${ }^{17}$ initially proposed a measurement method for the room gain and the voice support, which was later simplified by Pelegrin-Garcia. ${ }^{22}$ The present work uses the latter approach and refines its definition in terms of frequency weighting.

The two methods are based on the measurement of impulse responses (IRs) from the mouth to the ears of a dummy head, which are also referred to as oral-binaural room impulse responses (OBRIRs). ${ }^{35}$ An OBRIR shall be determined using a dummy head compliant to the standard ITU-T P.58, ${ }^{36}$ with a loudspeaker embedded in its mouth and microphones at its ears (at the position of the eardrum). Any acoustic measurement software can be used to determine the OBRIR, from the joint analysis of the excitation signal and the signals measured at the ears, when the excitation signal is reproduced through the mouth-loudspeaker. The dummy head shall be positioned with the mouth at a height of $1.5 \mathrm{~m}$ above the floor and at a distance of at least $1 \mathrm{~m}$ from other surfaces.

The method proposed by Brunskog et al. ${ }^{17}$ requires the measurement of two OBRIRs: One in an anechoic chamber and another one in the room of interest. The energy of the direct sound $E_{D}$ is extracted from the OBRIR measured in the anechoic chamber, and the total energy including direct sound and reflections $E_{D+R}$ is extracted from the OBRIR measured in the room of interest. Finally, the room gain is calculated assuming that $E_{D+R} \approx E_{D}+E_{R}$ (i.e., energy summation).

The alternative measurement method ${ }^{22}$ of room gain and voice support is carried out in a different way, although conceptually equivalent to that proposed by Brunskog et al. ${ }^{17}$ This method calculates the energy of the direct sound and the reflections from a single OBRIR in the room of interest. In this case, the direct sound $h_{D}(t)$ can be extracted from an OBRIR $h(t)$ by multiplying it with a window function $w(t)$,

$$
h_{D}(t)=h(t) w(t)
$$

because all the reflections will have a delay of at least $5.8 \mathrm{~ms}$ from the arrival time of the direct sound-the boundaries are at more than $1 \mathrm{~m}$. The window function is 


$$
w(t)= \begin{cases}1 & t<4.5 \mathrm{~ms} \\ 0.5+0.5 \cos \left[2 \pi\left(t-t_{0}\right) / T_{W}\right] & 4.5 \mathrm{~ms}<t<5.5 \mathrm{~ms} \\ 0 & t>5.5 \mathrm{~ms}\end{cases}
$$

with $t_{0}=4.5 \mathrm{~ms}$ as a time-shift parameter and $T_{W}=2 \mathrm{~ms}$ the period of the cosine function. The window function $w(t)$ is flat at unity from 0 to $4.5 \mathrm{~ms}$ and decays smoothly following half a period of the raised cosine function until it reaches a value of 0 at $5.5 \mathrm{~ms}$. The reflected sound $h_{R}(t)$ is obtained by multiplying the OBRIR by the complementary window function $1-w(t)$,

$$
h_{R}(t)=h(t)[1-w(t)] .
$$

An OBRIR and the windowing functions are shown in Fig. 1

The OBRIR and the signals of the direct sound and the reflected sound are filtered with six octave band filters $h_{F, i}(t)$, where $F$ stands for filter and $i$ indicates the band, which has a center frequency between $125 \mathrm{~Hz}(i=1)$ and $4 \mathrm{kHz}(i=6)$. The different bands are indicated in Table I. Therefore,

$$
\begin{aligned}
& h_{i}(t)=h(t) * h_{F, i}(t), \quad i=1, \ldots, 6, \\
& h_{D, i}(t)=h_{D}(t) * h_{F, i}(t), \quad i=1, \ldots, 6, \\
& h_{R, i}(t)=h_{R}(t) * h_{F, i}(t), \quad i=1, \ldots, 6,
\end{aligned}
$$

where * is the symbol of the convolution operator.

The energy levels of the direct sound $L_{D, i}$, the reflected sound $L_{R, i}$, and the OBRIR (direct + reflected sound) $L_{E, i}$ are calculated in octave bands as

$$
\begin{aligned}
L_{D, i} & =10 \log \frac{E_{D, i}}{E_{0}}=10 \log \frac{\int_{0}^{\infty}\left[h_{D, i}(t)\right]^{2} \mathrm{~d} t}{E_{0}}(\mathrm{~dB}), \\
i & =1, \ldots, 6, \\
L_{R, i} & =10 \log \frac{E_{R, i}}{E_{0}}=10 \log \frac{\int_{0}^{\infty}\left[h_{R, i}(t)\right]^{2} \mathrm{~d} t}{E_{0}}(\mathrm{~dB}), \\
i & =1, \ldots, 6,
\end{aligned}
$$

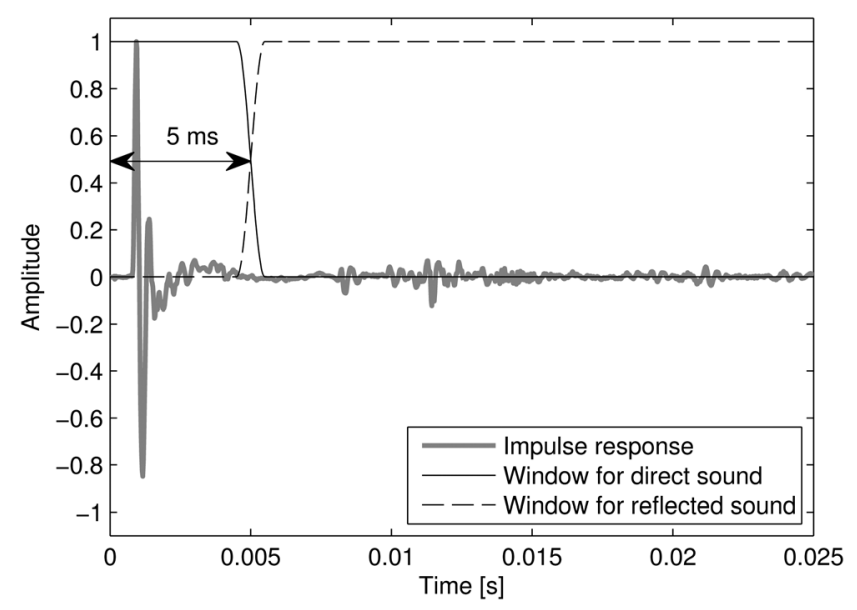

FIG. 1. Example of an OBRIR measured from the mouth and ears of a dummy head $h(t)$ and the windowing applied to extract the direct and the reflected sound (from Ref. 22).

$$
\begin{aligned}
L_{E, i} & =10 \log \frac{E_{D+R, i}}{E_{0}}=10 \log \frac{\int_{0}^{\infty}\left[h_{i}(t)\right]^{2} \mathrm{~d} t}{E_{0}}(\mathrm{~dB}), \\
i & =1, \ldots, 6
\end{aligned}
$$

where $E_{D, i}, E_{R, i}$, and $E_{D+R, i}$ are the energies of the direct sound, the reflected sound, and the OBRIR in the $i$ th octave band, respectively.

The room gain $G_{\mathrm{RG}, i}$ is calculated in each octave band as the difference between $L_{E, i}$ and $L_{D, i}$, whereas the voice support $S T_{V, i}$ is calculated in each octave band as the difference between $L_{R, i}$ and $L_{D, i}$.

In order to obtain single value descriptors of room gain and voice support, a frequency weighting is applied to the $S T_{V, i}$ values in the octave band. The reference spectrum is the typical speech level at the eardrum $L_{D, \text { ref }, i}$, indicated in Table I. This reference spectrum corresponds to a typical speech spectrum at $1 \mathrm{~m}$ in front of the speaker $^{37}$ corrected with the difference between the onaxis sound pressure level (SPL) at $1 \mathrm{~m}$ in front of a talker and the SPL at his ears in free-field. This difference was determined in an anechoic chamber by the simultaneous measurement of the SPL at the ears of a head and torso simulator (HATS) B\&K (Brüel \& Kjær Sound \& Vibration Measurement A/S; Nærum, Denmark) type 4128 with

\begin{tabular}{|c|c|c|c|c|c|c|}
\hline Octave band $i$ & 1 & 2 & 3 & 4 & 5 & 6 \\
\hline Center frequency $(\mathrm{Hz})$ & 125 & 250 & 500 & 1000 & 2000 & 4000 \\
\hline \multicolumn{7}{|c|}{ (1) Typical speech SPL on-axis at $1 \mathrm{~m}^{\mathrm{a}}$} \\
\hline$L_{D, \text { ref, } 1 m, i}(\mathrm{~dB})$ & 44.9 & 57.3 & 61.8 & 58.2 & 53.7 & 48.9 \\
\hline \multicolumn{7}{|c|}{ (2) Difference from SPL at eardrum (measured) } \\
\hline$L_{D, i}-L_{D, 1 m, i}(\mathrm{~dB})$ & 13.1 & 11.8 & 11.7 & 13.5 & 15.3 & 14.1 \\
\hline \multicolumn{7}{|c|}{ (3) Typical speech levels at the eardrum $=(1)+(2)$} \\
\hline$L_{D, \mathrm{ref}, i}(\mathrm{~dB})$ & 58.0 & 69.1 & 73.5 & 71.7 & 69.0 & 63.0 \\
\hline \multicolumn{7}{|c|}{ (4) Relation between $L_{W}$ and on-axis SPL at $1 \mathrm{~m}$} \\
\hline$L_{D, 1 m, i}-L_{W, i}(\mathrm{~dB})$ & -9.5 & -8.1 & -9.2 & -9.5 & -7.0 & -6.0 \\
\hline \multicolumn{7}{|c|}{ (5) Constant $K$ for model Eq. (23) $=(2)+(4)$} \\
\hline$K_{i}(\mathrm{~dB})$ & 3.6 & 3.7 & 2.5 & 4.0 & 8.3 & 8.1 \\
\hline \multicolumn{7}{|c|}{ (6) Directivity of human speech on downward direction } \\
\hline $\begin{array}{l}Q_{i}^{*} \\
\text { (7) Diffuse field HRTF }\end{array}$ & 0.95 & 0.78 & 0.79 & 0.60 & 0.21 & 0.25 \\
\hline$\Delta L_{\mathrm{HRTF}, i}(\mathrm{~dB})$ & 0 & 0 & 2 & 4 & 11 & 13 \\
\hline
\end{tabular}
mouth simulator, right ear simulator B\&K type 4158 , and left ear simulator B\&K type 4159 reproducing pink noise through its mouth in an anechoic chamber and of the SPL at $1 \mathrm{~m}$ in front of the mouth with a $1 / 2$ in. microphone B\&K type 4192.

The overall speech-weighted direct SPL $L_{D}$, the overall speech-weighted reflected SPL $L_{R}$, and the overall speechweighted total SPL $L_{E}$ are

TABLE I. Relevant frequency-dependent quantities used in the prediction model of voice support and its measurement.

${ }^{\mathrm{a}}$ Reference 37.

Pelegrín-García et al:: Measurement and prediction of voice support 


$$
\begin{aligned}
& L_{D}=10 \log \frac{\sum_{i=1}^{6} 10^{L_{D, \text { ref }, i} / 10}}{p_{0}^{2}}(\mathrm{~dB}), \\
& L_{R}=10 \log \frac{\sum_{i=1}^{6} 10^{L_{D, \text { re }, i}+\mathrm{ST}_{V, i} / 10}}{p_{0}^{2}}(\mathrm{~dB}), \\
& L_{E}=10 \log \frac{\sum_{i=1}^{6} 10^{L_{D, \text { ref }, i}+G_{\mathrm{RG}, i} / 10}}{p_{0}^{2}}(\mathrm{~dB}),
\end{aligned}
$$

with $p_{0}=20 \mu \mathrm{Pa}$ from which the overall speech-weighted room gain $G_{\mathrm{RG}}$ (or simply, room gain) is calculated as

$$
G_{\mathrm{RG}}=L_{E}-L_{D}=10 \log \frac{\sum_{i=1}^{6} 10^{L_{D, \text { ref }, i}+G_{\mathrm{RG}, i} / 10}}{\sum_{i=1}^{6} 10^{L_{D, \text { ref }, i} / 10}}(\mathrm{~dB})
$$

and the overall speech-weighted voice support $S T_{V}$ (or simply, voice support) as

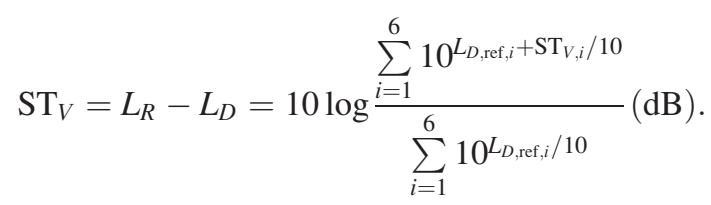

This process is illustrated and summarized in the block diagram in Fig. 2.

\section{Prediction model for voice support and room gain}

Using the definition of voice support in Eq. (3), a prediction model must account for the relation between the direct and the reflected sound at the ears, when the mouth acts as a source. To build this model, it is assumed that the measurement equipment is a HATS B\&K type 4128 with mouth simulator, right ear simulator B\&K type 4158 , and left ear simulator B\&K type 4159. The model predicts the position-averaged voice support in a room and is separated into the characterization of direct sound and the characterization of reflected sound.

\section{Characterization of the direct sound}

The propagation of the direct sound from the mouth to the ears cannot be assumed to be identical to that of a point source in free space, due to the diffraction of sound around the head and the filtering of the external ear. Therefore, a more empirical characterization is chosen, assuming that the airborne direct sound $L_{D, i}$ and the sound power level $L_{W, i}$ are related through

$$
L_{D, i}=L_{W, i}+K_{i}(\mathrm{~dB})
$$

where $K_{i}$ is a frequency-dependent constant with value

$$
K=\left(L_{D, i}-L_{D, 1 m, i}\right)+\left(L_{D, 1 m, i}-L_{W, i}\right)(\mathrm{dB}) .
$$

Here, the value of $K_{i}$ is decomposed into two quantities. The first quantity, $\left(L_{D, i}-L_{D, 1 m, i}\right)$, is the difference between the SPL at the ears of the dummy head and the SPL arises $1 \mathrm{~m}$ in front of its mouth in free-field. This difference was determined in an anechoic chamber by the simultaneous measurement of SPL at the ears of a HATS B\&K 4128, reproducing pink noise through its mouth in an anechoic chamber and of SPL at $1 \mathrm{~m}$ in front of the mouth with a $1 / 2 \mathrm{in}$. microphone B\&K type 4192 . The second quantity $\left(L_{D, 1 m, i}-L_{W, i}\right)$ is the difference between the on-axis SPL at $1 \mathrm{~m}$ in front of the mouth and the sound power level. It is determined from the speech directivity patterns measured by Chu and Warnock. ${ }^{38}$ The values of the two quantities and $K_{i}$ in the different octave bands are shown in Table I.

\section{Characterization of the reflected sound}

The reflected sound at the ears is characterized in this model by the diffuse reflections derived from the diffuse-field theory (which assumes the exponential decay of acoustic energy in an enclosure), the presence of a systematic floor reflection linked to the measurement method, and a correction for using a dummy head with microphones at its ears as a measurement device instead of an omnidirectional microphone.

The reflected SPL at a point in a room far from the source $L_{R, i}^{\prime}$, according to the diffuse-field theory, is related to the sound power level by means of

$$
L_{R, i}^{\prime}=L_{W, i}+10 \log \left(\frac{4}{R_{i}} S_{\text {ref }}\right)(\mathrm{dB}),
$$

where $R_{i}=S_{\text {tot }} \bar{\alpha}_{i} /\left(1-\bar{\alpha}_{i}\right)$ is sometimes called "room constant," $S_{\text {tot }}$ is the total surface area of the room and $\bar{\alpha}_{i}$ is the

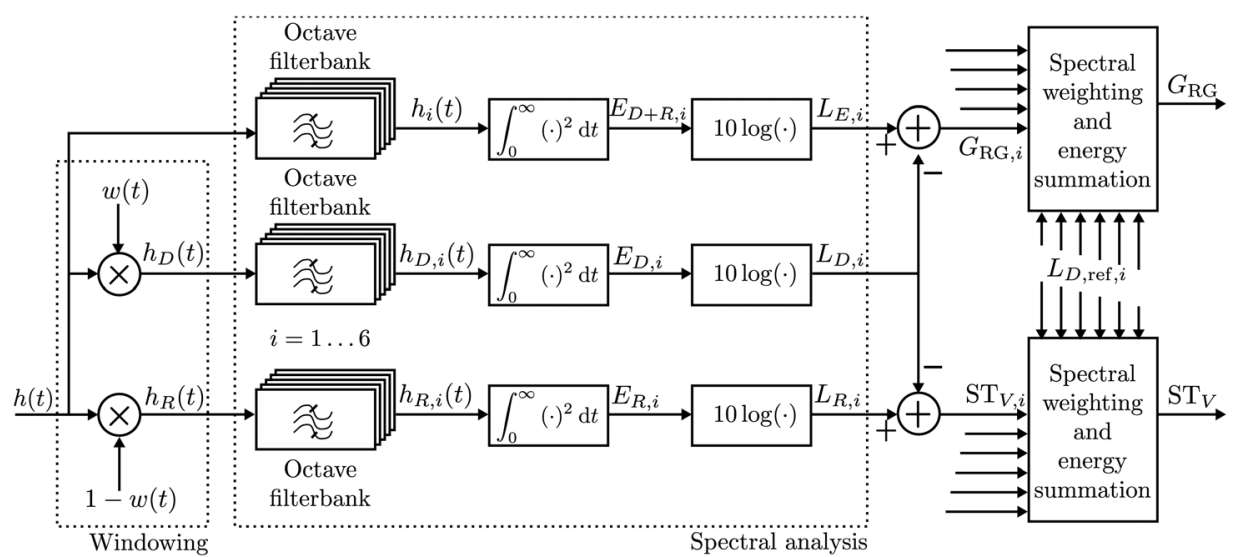

FIG. 2. Block diagram for the derivation of room gain and voice support from an oral-binaural room impulse response measurement $h(t)$. The bottom branch corresponds to the reflected sound, the middle one to the direct sound, and the top one to the total sound (direct and reflected). 
mean absorption coefficient, which is derived from the volume $V$ and the reverberation time $T_{60, i}$ measurements through Sabine's formula $\bar{\alpha}_{i}=4 \ln \left(10^{6}\right) V /\left(c S_{\text {tot }} T_{60, i}\right)$. The term $S_{\text {ref }}$ is the reference area

$$
S_{\mathrm{ref}}=\frac{W_{\mathrm{ref}} \rho_{0} c}{p_{0}^{2}}\left(\mathrm{~m}^{2}\right),
$$

where $W_{\text {ref }}=1 \mathrm{pW}$ is the reference power, $\rho_{0}$ is the density of the medium, and $c$ is the speed of sound. In air and normal conditions $\left(20{ }^{\circ} \mathrm{C}, 101.3 \mathrm{kPa}\right), \rho_{0} \approx 1.204 \mathrm{kgm}^{-3}, c \approx 343 \mathrm{~m} / \mathrm{s}$, and $S_{\text {ref }} \approx 1 \mathrm{~m}^{2}$.

Additionally, there is a floor reflection in all rooms, which can be regarded as systematic, because the floor is usually a highly reflective surface and the speaker is usually at an equal distance above the floor, whereas the other surfaces can be at different positions (the ceiling is at different heights in different rooms). The level of a reflection from the floor $L_{\mathrm{refl}, i}$ considering an image source from the mouth mirrored at the floor plane, would be

$$
L_{\mathrm{refl}, i}=L_{W, i}+10 \log \left(\frac{Q_{i}^{*}}{4 \pi(2 d)^{2}} S_{\mathrm{ref}}\right)(\mathrm{dB})
$$

at the position of the source and the ears (provided that the distance between both is much smaller than $2 d$ ). $Q_{i}^{*}$ is the directivity factor of speech in the downward direction (derived from Chu and Warnock ${ }^{38}$ ) and its frequency-dependent values are shown in Table I. The height $d$ is regarded as $1.5 \mathrm{~m}$, which is defined in the measurement method and corresponds to the mouth position of a standing female speaker.

Under these conditions, the expected reflected SPL at the position of the dummy head (without it disturbing the sound field) would be

$$
L_{R, i}^{\prime}=L_{W, i}+10 \log \left[\left(\frac{4}{R_{i}}+\frac{Q_{i}^{*}}{4 \pi(2 d)^{2}}\right) S_{\mathrm{ref}}\right](\mathrm{dB}) .
$$

Actually, the presence of the artificial head disturbs the sound field. Therefore, it is necessary to apply a correction term that relates the SPL at the measurement point when the equipment is present to the SPL at the same position in the absence of the equipment. In the case of the HATS, this correction corresponds to the definition of the head related transfer function (HRTF) and is notated as $\Delta L_{\mathrm{HRTF}, i}$. This magnitude is usually direction-dependent. As the reflected sound can arrive from many different directions, a direction averaged quantity - the diffuse field $\Delta L_{\mathrm{HRTF}, i}$ - given by the manufacturer $^{39}$ is used (see Table I).

The reflected sound level at the ears of the HATS is modeled as

$$
\begin{aligned}
L_{R, i}= & L_{W, i}+10 \log \left[\left(\frac{4}{R_{i}}+\frac{Q_{i}^{*}}{4 \pi(2 d)^{2}}\right) S_{\text {ref }}\right] \\
& +\Delta L_{\mathrm{HRTF}, i}(\mathrm{~dB}) .
\end{aligned}
$$

Finally, taking the difference between Eqs. (21) and (15) the frequency-dependent model for voice support is

$$
\begin{aligned}
\mathrm{ST}_{V, i}= & 10 \log \left[\left(\frac{4}{R_{i}}+\frac{Q_{i}^{*}}{4 \pi(2 d)^{2}}\right) S_{\mathrm{ref}}\right] \\
& +\Delta L_{\mathrm{HRTF}, i}-K_{i}(\mathrm{~dB})
\end{aligned}
$$

or in terms of directly measurable variables

$$
\begin{aligned}
\mathrm{ST}_{V, i}= & 10 \log \left[\left(\frac{c T_{i}}{\ln \left(10^{6}\right) V}-\frac{4}{S_{\mathrm{tot}}}+\frac{Q_{i}^{*}}{4 \pi(2 d)^{2}}\right) S_{\mathrm{ref}}\right] \\
& +\Delta L_{\mathrm{HRTF}, i}-K_{i}(\mathrm{~dB}) .
\end{aligned}
$$

The results from the individual bands should be weighted to obtain a single value by means of Eq. (14). Figure 3 shows an example set of curves for calculating $\mathrm{ST}_{V}$ from $V$ and $T_{60}$, assuming that the room has proportions 2.8:1.6:1 and the reverberation time has a flat frequency characteristic.

The room gain can be calculated from the voice support prediction model by using Eq. (4) and assuming that the total energy corresponds to the sum of the energies of the direct and the reflected sound, i.e., $E_{D+R} \approx E_{D}+E_{R}$.

\section{ACOUSTICS MEASUREMENTS IN CLASSROOMS}

Acoustics measurements were carried out in a total of 30 classrooms and sports halls from five different primary and secondary schools in southern Sweden, built during the 1970s. The schools were selected from a previous survey about voice problems among teachers and their relation to the teaching environment. ${ }^{12}$ The participation of the schools in this investigation was voluntary, and therefore it might be possible that only those schools with relatively good acoustic conditions volunteered. The schools were located in the suburbs of the city of Lund ( $\sim 100000$ inhabitants), away from main traffic routes and most of the classrooms had acoustically absorptive materials, mainly on the ceiling.

The objective parameters background noise level (BNL), reverberation time $T_{20}$, speech transmission index $\mathrm{STI}$, voice support $\mathrm{ST}_{V}$, and room gain $G_{\mathrm{RG}}$ were measured

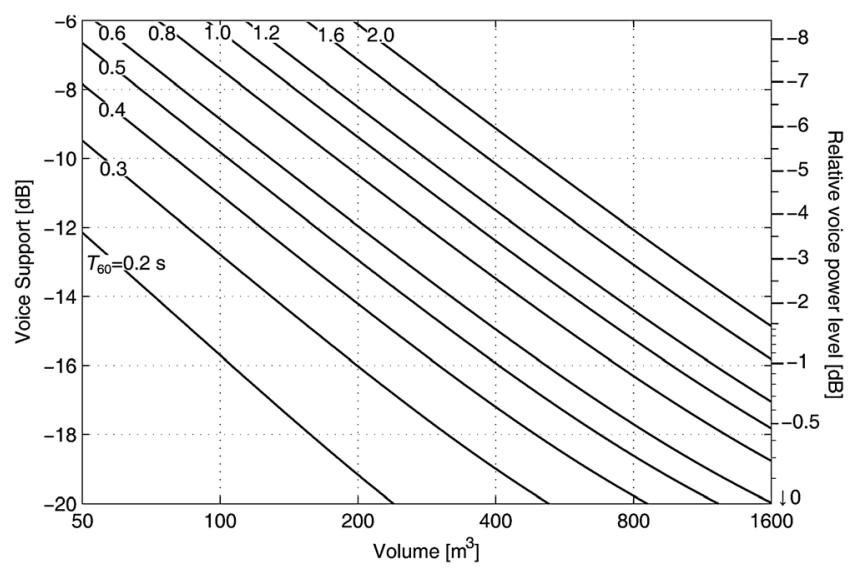

FIG. 3. Voice support versus room volume for a room of proportions 2.8:1.6:1 according to the predictions of the model in Eq. (23), for different values of reverberation time. The vertical axis on the right shows the average variations of voice power level experienced by talkers in these conditions, as measured by Brunskog et al. (Refs. 17 and 22) in the presence of low background noise levels $(<45 \mathrm{~dB})$. 
TABLE II. Average (standard deviation) dimensions and volumes of the measured school classrooms.

\begin{tabular}{lccccc}
\hline \hline Group size & $\begin{array}{c}\text { Number } \\
\text { of rooms }\end{array}$ & $W(\mathrm{~m})$ & $L(\mathrm{~m})$ & $H(\mathrm{~m})$ & Volume $\left(\mathrm{m}^{3}\right)$ \\
\hline Small & 3 & $4.5(0.8)$ & $3.3(0.8)$ & $2.7(0.2)$ & $40.6(18.3)$ \\
Medium & 24 & $8.9(1.7)$ & $7.0(1.0)$ & $2.8(0.2)$ & $180.2(61.6)$ \\
Large & 3 & $23.6(2.4)$ & $20.8(0.2)$ & $7.4(0.7)$ & $3614.3(77.0)$ \\
\hline \hline
\end{tabular}

in the 30 unoccupied but totally furnished rooms. The physical dimensions of the rooms are shown in Table II. According to the volume, the rooms were classified into three groups: small $\left(V<100 \mathrm{~m}^{3}\right)$, medium $\left(100<V<500 \mathrm{~m}^{3}\right)$, and large $\left(V>3500 \mathrm{~m}^{3}\right)$ classrooms. The rooms in the last group were sports halls where gymnastic lessons took place.

The measurements in the schools were performed after the lessons had finished, although there were some staff at school and some children in the playground. During the measurements, the doors and windows were closed and the ventilation systems were turned on.

\section{A. Measurement procedure}

\section{Background noise level measurements}

The A-weighted, $10 \mathrm{~s}$ equivalent BNL were measured in the empty classrooms using the $01 \mathrm{~dB}$ (01 dB-Metravib; Limonest Cedex, France) Symphonie system with two microphones of B\&K type 4192 at a height of $1.2 \mathrm{~m}$. Each microphone was placed at two different representative student positions in a classroom. Two measurements were taken at each position. Thus, for each classroom, a total of eight measurements (four positions, two repetitions) were carried out and averaged to obtain a unique BNL in the classroom.

\section{Measurements with an omnidirectional sound source}

The reverberation time $T_{20}$ and speech transmission index STI were derived from the measurements of the room IR using an omnidirectional sound source B\&K type 4295 "Omnisource." The source was placed at two different representative teaching positions, more than $1 \mathrm{~m}$ away from the walls, and with the radiating opening at a height of $1.6 \mathrm{~m}$ pointing upward. Two $1 / 2$ in. pressure-field microphones B\&K type 4192 were used as receivers and were placed close to student seats at a height of $1.2 \mathrm{~m}$ and more than $1 \mathrm{~m}$ away from the walls. The $01 \mathrm{~dB}$ Symphonie system, incorporating the MLS software module, was used to produce the measurement signal and send it to the loudspeaker via a power amplifier, acquire the signal from the microphones, calculate the room IR, and derive the parameters $T_{20}$ and STI. The $T_{20}$ was obtained by evaluating the backward integrated energy curve ${ }^{40}$ of the room IR in the decay interval from -5 to $-25 \mathrm{~dB}$. This range was chosen to unify the criteria in all classrooms, as it was the barely measurable range in the IR with the lowest signal-to-noise ratio [the earlydecay-time (EDT) is not given because it is influenced by the direct sound at the measurement positions close to the source]. A single value descriptor of the $T_{20}$ corresponding to the average of the octave bands between $500 \mathrm{~Hz}$ and $2 \mathrm{kHz} T_{500-2000}$ is given. The average standard deviation (SD) values of the signal-to-noise ratio of the IR measurements in the different classroom groups were $52 \mathrm{~dB}(4.1 \mathrm{~dB})$ in small classrooms, $46 \mathrm{~dB}(5.4 \mathrm{~dB})$ in middle-size classrooms, and $34 \mathrm{~dB}(5.4 \mathrm{~dB})$ in large classrooms.

\section{Measurements with a dummy head}

The room gain and the voice support were determined from the measurement of OBRIRs in the empty classrooms with the method detailed in Sec. II B. For this purpose, a HATS B\&K type 4128 was used. The HATS included a loudspeaker at its mouth and microphones at its ears. The HATS was placed at two representative teaching positions, with the mouth at a height of $1.5 \mathrm{~m}$, and more than $1 \mathrm{~m}$ away from reflecting surfaces. The $01 \mathrm{~dB}$ Symphonie system was used to produce the excitation signal and determine the OBRIR from the measured signal at the microphones. For each classroom, the room gain and the voice support values corresponded to the average of four measurements (two ears at two different positions).

\section{B. Results of the measurements}

\section{Correlation between parameters}

The correlation coefficients between the measurements of the magnitudes $V, \log (V), \mathrm{BNL}, T_{500-2000}, \mathrm{STI}, G_{\mathrm{RG}}$, and $\mathrm{ST}_{V}$ are shown in Table III. The BNL has very low correlation with all the other parameters, because it is not determined from physical properties of the room, but depends on different noise sources, primarily from installations inside the room, and secondarily from other external noise sources (traffic noise, people passing by the corridors, and pupils on the playground). The reverberation time is correlated to the volume and negatively correlated to the STI. The voice support is strongly negatively correlated to the logarithm of the volume, as expected from the prediction model in Eq. (23) and Fig. 3. The same figure also points out a negative correlation between voice support and reverberation time $(-0.70$ in the measurements). The presence of some high correlation coefficients is largely caused by the large measured range of volumes and most of the other parameters of

TABLE III. Correlation coefficients indicating the strength of the linear dependence between pairs of variables. ${ }^{\text {a }}$

\begin{tabular}{lccccccc}
\hline \hline & $V$ & $\log V$ & $\mathrm{BNL}$ & $T_{500-2000}$ & $\mathrm{STI}$ & $G_{\mathrm{RG}}$ & $\mathrm{ST}_{V}$ \\
\hline$V$ & 1.00 & $\mathbf{0 . 9 1}$ & - & $\mathbf{0 . 9 7}$ & $\mathbf{- 0 . 8 1}$ & -0.50 & $\mathbf{- 0 . 7 5}$ \\
$\log V$ & & 1.00 & - & $\mathbf{0 . 9 1}$ & $\mathbf{- 0 . 5 7}$ & $\mathbf{- 0 . 7 5}$ & $\mathbf{- 0 . 8 7}$ \\
$\mathrm{BNL}$ & & & 1.00 & - & - & - & - \\
$T_{500-2 k}$ & & & & 1.00 & $\mathbf{- 0 . 8 9}$ & -0.49 & $\mathbf{- 0 . 7 0}$ \\
$\mathrm{STI}$ & & & & & 1.00 & $\mathbf{0 . 5 5}$ & $\mathbf{0 . 6 8}$ \\
$G_{\mathrm{RG}}$ & & & & & & 1.00 & $\mathbf{0 . 9 4}$ \\
$\mathrm{ST}_{V}$ & & & & & & & 1.00 \\
\hline \hline
\end{tabular}

${ }^{a}$ Only correlation coefficients with $p<0.05$ are shown. Correlation coefficients with $<0.001$ are marked as bold and coefficients with $0.05<p<0.001$ are marked in italics. 
TABLE IV. Mean, standard deviation (SD) of the mean, and maximum background noise levels (BNLs) measured in the classrooms in the different octave bands and overall A-weighted values.

\begin{tabular}{lccccccc}
\hline $\begin{array}{l}\text { Octave band center } \\
\text { frequency (Hz) }\end{array}$ & 125 & 250 & 500 & 1000 & 2000 & 4000 & A-weighted \\
\hline Small classrooms & & & & & & & \\
Mean BNL (dB) & 38.3 & 32.4 & 28.2 & 26.1 & 22.3 & 19.4 & 32.3 \\
SD (dB) & 9.5 & 8.2 & 7.3 & 5.6 & 4.5 & 2.1 & 5.7 \\
Maximum BNL (dB) & 48.8 & 39.3 & 34.5 & 32.6 & 27.5 & 21.3 & 38.5 \\
Medium classrooms & & & & & & & \\
Mean BNL (dB) & 40.2 & 33.7 & 27.8 & 24.4 & 22.7 & 19.9 & 32.7 \\
SD (dB) & 5.5 & 4.8 & 6.5 & 5.5 & 5.4 & 4.6 & 5.1 \\
Maximum BNL (dB) & 53.4 & 43.6 & 43.7 & 40.1 & 37.3 & 32.4 & 43.5 \\
Large classrooms & & & & & & & \\
Mean BNL (dB) & 45.1 & 37.9 & 33.5 & 32.0 & 28.3 & 21.9 & 37.6 \\
SD (dB) & 6.3 & 7.7 & 7.0 & 5.5 & 2.9 & 1.4 & 5.7 \\
Maximum BNL (dB) & 51.5 & 46.2 & 41.1 & 37.4 & 30.1 & 23.2 & 43.5 \\
\hline \hline
\end{tabular}

the classrooms. The correlation between $V$ and $T_{500-2000}$ is 0.97 , due to the large difference between the volumes in the sports halls and the rest of the classrooms (see Table II) and the similarity of reverberation times and placement of absorptive materials within each group.

\section{Background noise levels}

The averaged 10-s equivalent background noise levels (A-weighted and in one octave frequency bands, obtained from the average of eight measurements) of each classroom were taken into account to report in Table IV the mean, SD, and maximum values of BNL within each group of classrooms (small, medium, large). Even though it is not explicitly shown, $73.3 \%$ of the classrooms had BNLs lower than $35 \mathrm{~dB}$, another $13.3 \%$ between 35 and $40 \mathrm{~dB}$, and the remaining $13.3 \%$ of the measurements were between 40 and $45 \mathrm{~dB}$. In most of the cases, the noise sources corresponded to the ventilation systems, and in a few cases, the background noise was affected by external sources, such as people passing by the corridor, pupils on the playground, and traffic. The background noise levels were similar for all room sizes, although the overall level in the large rooms was slightly higher than in smaller rooms. In all cases, low frequency noise was markedly dominating. This is an indication that the sources, in most of the cases, were, in fact, the machinery of the ven-

TABLE V. Reverberation times $\left(T_{20}\right)$ measured in the classrooms.

\begin{tabular}{lccccccc}
\hline \hline $\begin{array}{l}\text { Octave band center } \\
\text { frequency }(\mathrm{Hz})\end{array}$ & 125 & 250 & 500 & 1000 & 2000 & 4000 & $\begin{array}{c}\text { Average } \\
500-2000\end{array}$ \\
\hline $\begin{array}{l}\text { Small classrooms } \\
\text { Mean } T_{20}(\mathrm{~s})\end{array}$ & 0.59 & 0.39 & 0.32 & 0.34 & 0.35 & 0.34 & 0.33 \\
$\mathrm{SD}(\mathrm{s})$ & 0.42 & 0.14 & 0.04 & 0.05 & 0.05 & 0.02 & 0.05 \\
Medium classrooms & & & & & & & \\
Mean $T_{20}(\mathrm{~s})$ & 0.72 & 0.53 & 0.45 & 0.47 & 0.47 & 0.44 & 0.46 \\
$\mathrm{SD}(\mathrm{s})$ & 0.33 & 0.17 & 0.08 & 0.08 & 0.07 & 0.07 & 0.08 \\
Large classrooms & & & & & & & \\
Mean $T_{20}(\mathrm{~s})$ & 1.46 & 1.58 & 1.59 & 1.55 & 1.35 & 1.04 & 1.57 \\
$\mathrm{SD}(\mathrm{s})$ & 0.24 & 0.35 & 0.29 & 0.18 & 0.07 & 0.07 & 0.23 \\
\hline \hline
\end{tabular}

tilation systems, or external noise that leaked into the room due to the usually poor insulation performance of walls, doors, and windows at low frequencies.

\section{Reverberation time}

The mean reverberation times (in octave bands and $500 \mathrm{~Hz}-2 \mathrm{kHz}$ average) and their standard deviation are shown in Table V. An 81.5\% (22 out of 27) of the small and medium classrooms had reverberation times lower than $0.5 \mathrm{~s}$, and the remaining $18.5 \%$ were between 0.5 and $0.6 \mathrm{~s}$. In the sports halls, $T_{500-2000}$ was between 1.4 and $1.8 \mathrm{~s}$.

\section{Speech transmission index}

The average (standard deviation) measured STI in unoccupied classrooms, with a negligible effect of background noise, was $0.80(0.02)$ in small classrooms, $0.75(0.03)$ in medium classrooms, and $0.63(0.02)$ in large classrooms. The spread of STI among rooms, indicated by the standard deviation, was similar in all of the three classroom groups. The small classrooms had the highest STI, which is in the category of "excellent." 41 The medium classrooms had an average STI rating that is between "good" and "excellent," and the sports halls had a STI rating of "good." These ratings, however, are likely to become lower in occupied conditions due to an increase in activity noise, even though the reverberation time decreases.

\section{Voice support and room gain}

The mean and standard deviation of $\mathrm{ST}_{V}$ and $G_{\mathrm{RG}}$ in the octave bands between $125 \mathrm{~Hz}$ and $4 \mathrm{kHz}$ measured in the classrooms is shown in Table VI. The frequency characteristics of $\mathrm{ST}_{V}$ and $G_{\mathrm{RG}}$ are similar for small and medium classrooms, with an increase of the values at high frequencies. The only difference between the two classroom groups is that the small classrooms have a slightly higher overall value. The large classrooms (sports halls) have an overall lower value and, in addition, the frequency characteristic is

TABLE VI. Frequency band values and overall speech-weighted voice support $\left(\mathrm{ST}_{V}\right)$ and room gain $\left(G_{\mathrm{RG}}\right)$ measured in the classrooms.

\begin{tabular}{|c|c|c|c|c|c|c|c|}
\hline $\begin{array}{l}\text { Octave band center } \\
\text { frequency }(\mathrm{Hz})\end{array}$ & 125 & 250 & 500 & 1000 & 2000 & 4000 & $\begin{array}{l}\text { Speech- } \\
\text { weighted }\end{array}$ \\
\hline \multicolumn{8}{|l|}{ Small classrooms } \\
\hline Mean $\mathrm{ST}_{V}(\mathrm{~dB})$ & -9.4 & -11.1 & -9.5 & -7.6 & -6.4 & -4.6 & -5.6 \\
\hline $\mathrm{SD}(\mathrm{dB})$ & 0.46 & 0.81 & 0.91 & 0.38 & 0.72 & 1.04 & 0.78 \\
\hline Mean $G_{\mathrm{RG}}(\mathrm{dB})$ & 0.50 & 0.33 & 0.47 & 0.70 & 0.91 & 1.31 & 1.06 \\
\hline $\mathrm{SD}(\mathrm{dB})$ & 0.02 & 0.06 & 0.09 & 0.06 & 0.13 & 0.25 & 0.16 \\
\hline \multicolumn{8}{|l|}{ Medium classrooms } \\
\hline Mean $\mathrm{ST}_{V}(\mathrm{~dB})$ & -12.1 & -13.9 & -13.5 & -11.6 & -10.9 & -9.1 & -10.2 \\
\hline $\mathrm{SD}(\mathrm{dB})$ & 1.46 & 1.27 & 1.43 & 1.68 & 1.75 & 1.52 & 1.58 \\
\hline Mean $G_{\mathrm{RG}}(\mathrm{dB})$ & 0.28 & 0.18 & 0.20 & 0.32 & 0.37 & 0.54 & 0.42 \\
\hline $\mathrm{SD}(\mathrm{dB})$ & 0.10 & 0.06 & 0.07 & 0.13 & 0.16 & 0.19 & 0.16 \\
\hline \multicolumn{8}{|l|}{ Large classrooms } \\
\hline Mean $\mathrm{ST}_{V}(\mathrm{~dB})$ & -10.8 & -16.0 & -18.2 & -19.1 & -19.5 & -19.4 & -18.8 \\
\hline $\mathrm{SD}(\mathrm{dB})$ & 1.56 & 1.91 & 0.92 & 1.31 & 1.40 & 1.31 & 1.01 \\
\hline Mean $G_{\mathrm{RG}}(\mathrm{dB})$ & 0.36 & 0.12 & 0.07 & 0.06 & 0.05 & 0.06 & 0.058 \\
\hline $\mathrm{SD}(\mathrm{dB})$ & 0.14 & 0.06 & 0.01 & 0.02 & 0.02 & 0.02 & 0.01 \\
\hline
\end{tabular}

Pelegrín-García et al:: Measurement and prediction of voice support 
qualitatively different, because the low frequencies are predominant. This indicates that these large rooms do not reflect efficiently the high frequencies of a speaker. The spread of $\mathrm{ST}_{V}$ among rooms does not depend on the frequency band, because the standard deviation does not present a frequency-dependent pattern in the different classroom groups.

\section{VALIDATION OF THE PREDICTION MODEL FOR VOICE SUPPORT}

\section{A. Parameters used to assess the prediction model}

The prediction model for $\mathrm{ST}_{V}$ in Eq. (23) was evaluated for each classrooms in octave bands by using the frequencydependent measured values of $T_{20}$, along with the volume $V$ and total surface area $S_{\text {tot. }}$ In addition, a broadband value (speech-weighted $\mathrm{ST}_{V}$ ) was calculated from the frequencyband values using Eq. (14).

The prediction model was assessed by comparing the measured voice support values $\mathrm{ST}_{V \text {,meas }}$ and the predicted voice support values $\mathrm{ST}_{V \text {,pred. }}$ In each octave band (or overall speech-weighted), a regression line of the type $\overline{\mathrm{ST}}_{V \text {,pred }}$ $=a \mathrm{ST}_{V \text {,meas }}+b$ was calculated, where $\overline{\mathrm{ST}}_{V \text {,pred }}$ is the regressor for the predicted values of voice support and $a$ and $b$ are the coefficients of the regression line. An unbiased model would result if $a=1$ and $b=0$, i.e., if the regression line of the predicted values is on top of the measurements $\left(\overline{\mathrm{ST}}_{V \text {,pred }}\right.$ $\left.=\mathrm{ST}_{V \text {,meas }}\right)$.

The goodness-of-fit of the prediction model was evaluated with three parameters: (a) the coefficient of determina- tion $R^{2}$ of the linear regression model for the measured versus predicted values, (b) the residual deviation $\sigma_{\epsilon}$ of the predicted values from this regression line, and (c) the deviation $\sigma_{T}$ of the predicted values from an unbiased prediction, which is a measure of the bias in the prediction

$$
\begin{aligned}
\sigma_{\epsilon}^{2} & =\frac{1}{N-2} \sum_{i=1}^{N}\left(\mathrm{ST}_{V, \text { pred }}-\overline{\mathrm{ST}}_{V, \text { pred }}\right)^{2}, \\
\sigma_{T}^{2} & =\frac{1}{N} \sum_{i=1}^{N}\left(\mathrm{ST}_{V, \text { pred }}-\mathrm{ST}_{V, \text { meas }}\right)^{2} .
\end{aligned}
$$

\section{B. Correspondence between measured and predicted values}

The comparison between the measured and the predicted values of $\mathrm{ST}_{V}$ in the octave bands between $125 \mathrm{~Hz}$ and $4 \mathrm{kHz}$ is shown in Fig. 4. The most accurate predictions were found in the most important bands for speech (between $500 \mathrm{~Hz}$ and $2 \mathrm{kHz}$ ). In these bands, $R^{2}$ was at least 0.8 , the residual deviation was not higher than $1.2 \mathrm{~dB}$, and the bias or deviation from the unbiased prediction was lower than $2 \mathrm{~dB}$. The prediction for the $125 \mathrm{~Hz}$ band had a large uncertainty, shown by the low value of $R^{2}(0.18)$, and large residual deviation $(3.3 \mathrm{~dB})$ and bias $(4.3 \mathrm{~dB})$.

The speech-weighted $\mathrm{ST}_{V}$ predictions are plotted in Fig. 5 as a function of the measured $\mathrm{ST}_{V}$ values. The regression line relating measurements and predictions had a slope of 1 and an offset of $0.36 \mathrm{~dB}$. The $R^{2}$ was 0.84 , the residual error was $1.1 \mathrm{~dB}$ and the bias was $1.2 \mathrm{~dB}$.
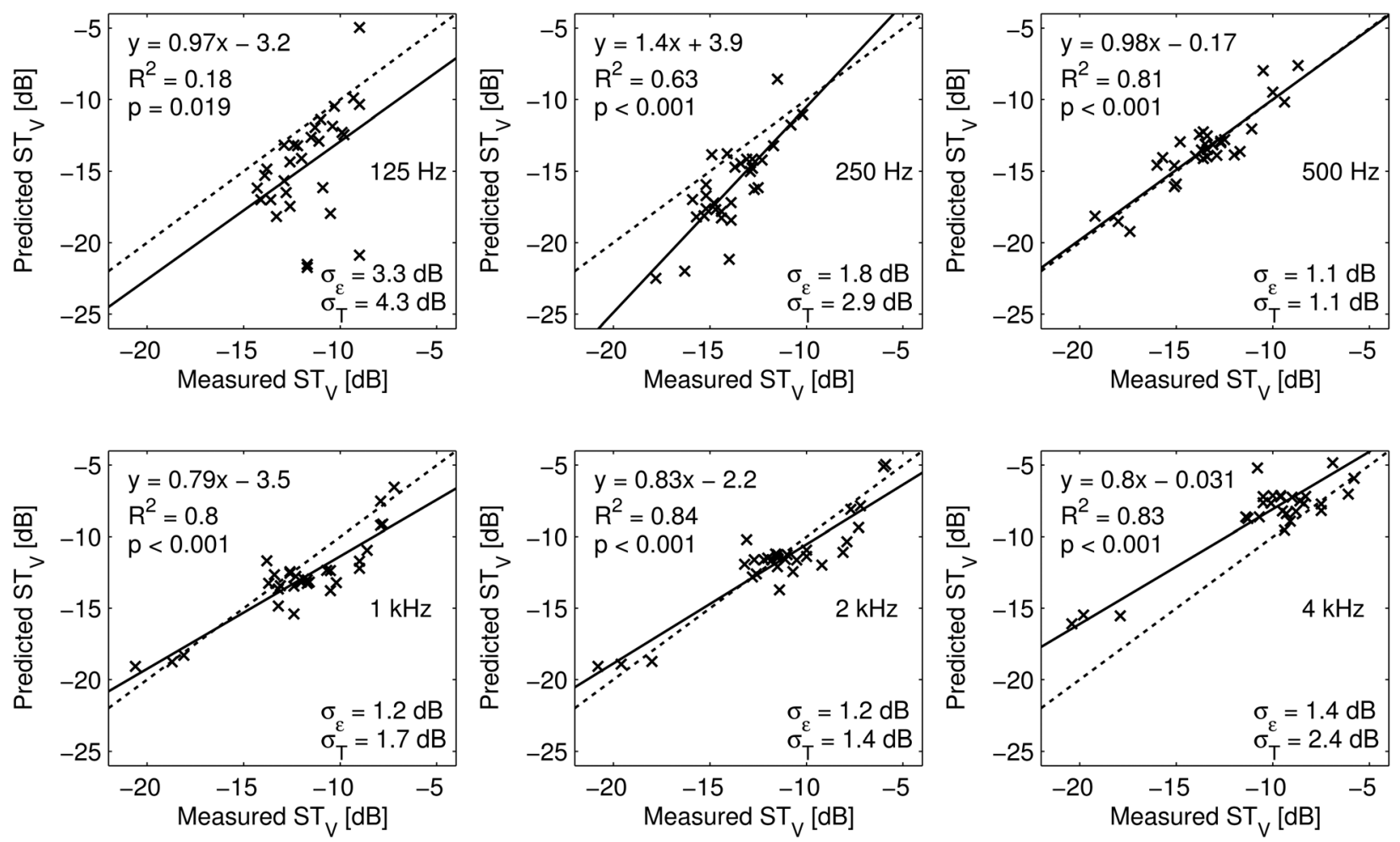

FIG. 4. Predicted versus measured values of voice support in frequency bands. The solid lines show the regression lines for the predictions and the dotted lines indicate the unbiased prediction lines. 


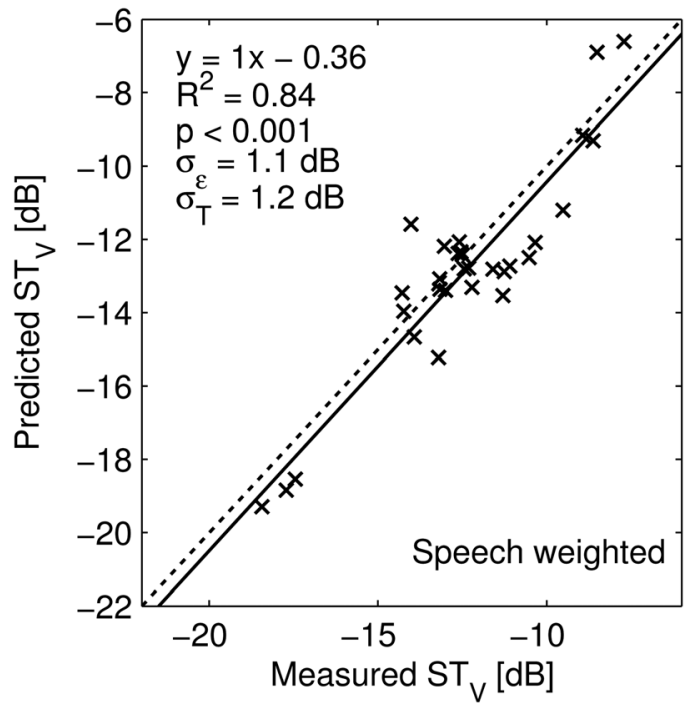

FIG. 5. Predicted versus measured speech-weighted overall values of voice support. The solid line shows the regression line for the predictions and the dotted line indicates the unbiased prediction line.

\section{DISCUSSION}

The acoustic properties of school classrooms described in the results section correspond to typical primary and secondary schools in southern Sweden built during the 1970s. The background noise levels in almost three-fourths of the small- and medium-sized classrooms were below $35 \mathrm{dBA}-$ which is the maximum acceptable value of different guidelines, e.g., the standard ANSI/ASA S12.60-2010/Part $1^{31}$ in the United States, the Building Bulletin $93^{32}$ in the United Kingdom, or the guidelines from the World Health Organisation. ${ }^{34}$ The background noise levels in the remaining fourth of classrooms were half below $40 \mathrm{~dB}$ and half between 40 and $45 \mathrm{~dB}$. The average value was $32.6 \mathrm{~dB}$, which is lower than the $45-48 \mathrm{~dB}$ reported by Shield and Dockrell ${ }^{1}$ in their review from several surveys on empty classrooms (without acoustical treatment).

In small- and medium-sized classrooms, the reverberation times did not exceed $0.6 \mathrm{~s}$, in fulfillment of different guidelines of classroom acoustic design. ${ }^{31,32,34}$ Reverberation times and BNLs were within the recommended values in most of the cases. This seems to be reflected in the nonproblematic perception of classroom acoustics by teachers without voice problems in schools of the same region in Sweden. ${ }^{12}$ The Swedish standard for acoustic conditions in classrooms $^{33}$ is more strict, requiring reverberation times below $0.5 \mathrm{~s}$ for the octave frequency bands above $250 \mathrm{~Hz}$ and below $0.6 \mathrm{~s}$ at $125 \mathrm{~Hz}$, which only a few of the classrooms met.

The STI measured in classrooms with high signalto-noise ratios was higher than 0.6 in all cases, even in the sports halls. However, the STI ratings and the speech intelligibility with ongoing activity, specially in the sports halls, will be lower than predicted, due to an actual lower signalto-noise ratio under these conditions.

The prediction model for $\mathrm{ST}_{V}$ has been derived theoretically and it has been assessed by comparing its predictions with actual $\mathrm{ST}_{V}$ measurements. There is a slight bias in the prediction, as the regression line of measured versus predicted $\mathrm{ST}_{V}$ is not $\overline{\mathrm{ST}}_{V \text {,pred }}=\mathrm{ST}_{V \text {, meas }}$, but $\overline{\mathrm{ST}}_{V \text {, pred }}=1.0 \mathrm{ST}_{V \text {, meas }}$ $-0.36 \mathrm{~dB}$ (see Fig. 5). This bias results in a deviation of $1.2 \mathrm{~dB}$ from the actual values, slightly higher than the residual deviation $(1.1 \mathrm{~dB})$ of the regression model. Taking into account that the measurement data set has not been used to derive the model, the predictions are reasonably accurate.

In the range of medium-sized classrooms (with volumes $\left.100<V<250 \mathrm{~m}^{3}\right), G_{\mathrm{RG}}$ is in the range between 0.2 and $0.5 \mathrm{~dB}$, whereas $\mathrm{ST}_{V}$ is in the range between -14 and $-9 \mathrm{~dB}$. There is some spread of data in this range, as seen in Fig. 5 where, as an example, there are two data points or classrooms for which the model predicts a similar average $\mathrm{ST}_{V}$ of $\sim-11.5 \mathrm{~dB}$ for both of them, whereas the actually measured $\mathrm{ST}_{V}$ 's are -14 and $-9.5 \mathrm{~dB}$. Thus, measured $\mathrm{ST}_{V}$ values can deviate as much as $\pm 3 \mathrm{~dB}$ from the predicted value. The $\mathrm{ST}_{V}$ is influenced by the early reflections which cannot be accurately represented with a statistical model such as the one in Eq. (23), which only aims at predicting a position-averaged value.

The voice support, analogous to the objective support in concert halls, is not a stand-alone parameter to design classroom acoustics (other magnitudes, like $T_{60}$, STI, and BNL, should be taken into account as well). It is a magnitude related to the additional vocal load that teachers experience when speaking in a classroom due to the acoustic conditions. The right-hand axis in Fig. 3 shows the voice power level variations expected from teachers speaking in different rooms in the presence of low background noise levels $(<45 \mathrm{~dB})$, first reported by Brunskog et al. ${ }^{17}$ This axis has been derived from the empirical models of Eq. (12) in Ref. 23 for the range $\mathrm{ST}_{V}<-14.5 \mathrm{~dB}$ and Eq. (13) in Ref. 23 for the range $\mathrm{ST}_{V}>-14.5 \mathrm{~dB}$. As an example, one can evaluate the effect of reducing the reverberation time in a room of $200 \mathrm{~m}^{3}$ from 1 to $0.5 \mathrm{~s}$ in terms of voice power level demands. In Fig. 3, the intersection between $V=200 \mathrm{~m}^{3}$ and $T_{60}=1.0 \mathrm{~s}$ occurs for $\mathrm{ST}_{V} \approx-9.5 \mathrm{~dB}$, which corresponds to a relative voice power level of $\sim-5.7 \mathrm{~dB}$. The intersection between $V=200 \mathrm{~m}^{3}$ and $T_{60}=0.5 \mathrm{~s}$ occurs for $\mathrm{ST}_{V} \approx$ $-12.8 \mathrm{~dB}$, which corresponds to a relative voice power level of $\sim-3.0 \mathrm{~dB}$. Therefore, the reduction of reverberation time in a room of $200 \mathrm{~m}^{3}$ from 1.0 to $0.5 \mathrm{~s}$ would result in an increase of voice power level of $-3.0-(-5.7)=+2.7 \mathrm{~dB}$. However, higher reverberation times would increase the activity noise level of students, as pointed out by Hodgson et al., ${ }^{19}$ which would also make the teacher raise his voice power level due to the Lombard effect.

There is not enough scientific evidence to establish a definite range of recommended values of $\mathrm{ST}_{V}$, but the range between -14 and $-9 \mathrm{~dB}$ (or a range of room gain between 0.2 and $0.5 \mathrm{~dB}$ ) obtained in most of the medium-sized classrooms seems adequate, as $T_{20}$ and STI fulfilled the recommendations without the rooms being too damped.

Using high values of room gain or voice support should not compromise the speech intelligibility in classrooms. According to the quality indices used by Nijs and Rychtarikova, "good" speech intelligibility is achieved for an early-late ratio $C_{50}$ not lower than $1.5 \mathrm{~dB}$ - which using a diffuse-field model ${ }^{11}$ is equivalent to a reverberation time 
not higher than $0.8 \mathrm{~s}$. Therefore, the maximum average voice support in classrooms, as a function of the volume, is given by the line $T_{60}=0.8 \mathrm{~s}$ in Fig. 3. Such a reverberation time limits the average $\mathrm{ST}_{V}$ to a maximum value of $-7.5 \mathrm{~dB}$ in rooms of $100 \mathrm{~m}^{3},-10.5 \mathrm{~dB}$ in rooms of $200 \mathrm{~m}^{3}$, and $-13.5 \mathrm{~dB}$ in rooms of $400 \mathrm{~m}^{3}$ in order to preserve speech intelligibility.

\section{CONCLUSIONS}

The present paper has measured and provided a reference set for voice support and room gain values, which are important parameters to assess the vocal effort required to speak in a room. The voice support in classrooms of good acoustical quality, with volumes between 100 and $250 \mathrm{~m}^{3}$, has been found to be in the range between -14 and $-9 \mathrm{~dB}$, and the room gain in the range between 0.2 and $0.5 \mathrm{~dB}$.

A model, derived from the diffuse field theory, has been developed to predict average values of voice support in classrooms and average variations in voice power level experienced by teachers in different rooms in the presence of low background noise levels. The model is based on geometrical room properties of volume, total surface area, and reverberation time. It points out necessary geometrical restrictions in rooms to obtain good acoustic conditions both for a listener (in terms of reverberation time) and for a speaker (in terms of voice support). The model describes the present voice support measurements in classrooms with a coefficient of determination of 0.84 and a standard deviation of $1.2 \mathrm{~dB}$.

\section{ACKNOWLEDGMENTS}

This research has been funded by the Swedish organization AFA Försäkring as a part of the project "Speaker's comfort and voice health in classrooms." Tobias Olesen from DTU has given some important feedback to improve the content of the paper. The authors would like to express their gratitude to all the schools and staff who made these measurements possible.

${ }^{1}$ B. Shield and J. Dockrell, "The effects of noise on children at school: a review," Build. Acoust. 10, 97-116 (2003).

${ }^{2}$ B. Shield and J. Dockrell, "The effects of environmental and classroom noise on the academic attainments of primary school children," J. Acoust. Soc. Am. 123, 133-144 (2008).

${ }^{3}$ M. Klatte, M. Meis, H. Sukowski, and A. Schick, "Effects of irrelevant speech and traffic noise on speech perception and cognitive performance in elementary school children," Noise Health 9, 64-73 (2007).

${ }^{4}$ A. Neuman and I. Hochberg, "Children's perception of speech in reverberation," J. Acoust. Soc. Am. 73, 2145-2149 (1983).

${ }^{5}$ J. Bradley, H. Sato, and M. Picard, "On the importance of early reflections for speech in rooms," J. Acoust. Soc. Am. 113, 3233-3244 (2003).

${ }^{6}$ A. Neuman, M. Wroblewski, J. Hajicek, and A. Rubinstein, "Combined effects of noise and reverberation on speech recognition performance of normal-hearing children and adults," Ear Hear. 31, 336-344 (2010).

${ }^{7}$ J. Bradley, "Speech intelligibility studies in classrooms," J. Acoust. Soc. Am. 80, 846-854 (1986).

${ }^{8}$ J. Bradley, R. Reich, and S. Norcross, "On the combined effects of signalto-noise ratio and room acoustics on speech intelligibility," J. Acoust. Soc. Am. 106, 1820-1828 (1999).

${ }^{9}$ M. Klatte, T. Lachmann, and M. Meis, "Effects of noise and reverberation on speech perception and listening comprehension of children and adults in a classroom-like setting," Noise Health 12, 270-282 (2010).

${ }^{10} \mathrm{~J}$. Bradley, "Predictors of speech intelligibility in rooms," J. Acoust. Soc. Am. 80, 837-845 (1986).
${ }^{11}$ S. R. Bistafa and J. S. Bradley, "Reverberation time and maximum background-noise level for classrooms from a comparative study of speech intelligibility metrics," J. Acoust. Soc. Am. 107, 861-875 (2000).

${ }^{12}$ V. Lyberg-Åhlander, R. Rydell, and A. Löfqvist, "Speaker's comfort in teaching environments: Voice problems in Swedish teaching staff," J. Voice 25, 430-440 (2011).

${ }^{13}$ N. Roy, R. Merrill, S. Thibeault, R. Parsa, S. Gray, and E. Smith, "Prevalence of voice disorders in teachers and the general population," J. Speech Lang. Hear. Res. 47, 281-293 (2004).

${ }^{14} \mathrm{E}$. Vilkman, "Voice problems at work: A challenge for occupational safety and health arrangement," Folia Phoniatr. Logo. 52, 120-125 (2000).

${ }^{15} \mathrm{E}$. Lombard, "Le signe de l'elevation de la voix (The sign of the elevation of the voice)," Ann. Maladies Oreille, Larynx, Nez, Pharynx 37, 101-119 (1911).

${ }^{16} \mathrm{H}$. Lazarus, "Prediction of verbal communication in noise-a review: Part 1" Appl. Acoust. 19, 439-463 (1986).

${ }^{17}$ J. Brunskog, A. Gade, G. P. Ballester, and L. R. Calbo, "Increase in voice level and speaker comfort in lecture rooms," J. Acoust. Soc. Am. 125, 2072-2082 (2009).

${ }^{18}$ D. Pelegrin-Garcia, B. Smits, J. Brunskog, and C.-H. Jeong, "Vocal effort with changing talker-to-listener distance in different acoustic environments," J. Acoust. Soc. Am. 129, 1981-1990 (2011).

${ }^{19}$ M. Hodgson, R. Rempel, and S. Kennedy, "Measurement and prediction of typical speech and background-noise levels in university classrooms during lectures," J. Acoust. Soc. Am. 105, 226-233 (1999).

${ }^{20}$ M. Kob, G. Behler, A. Kamprolf, O. Goldschmidt, and C. NeuschaeferRube, "Experimental investigations of the influence of room acoustics on the teachers voice," Acoust. Sci. Tech. 29, 86-94 (2008).

${ }^{21}$ L. Nijs and M. Rychtarikova, "Calculating the optimum reverberation time and absorption coefficient for good speech intelligibility in classroom design using U50" Acta Acust. Acust. 97, 93-102 (2011).

${ }^{22}$ D. Pelegrin-Garcia, "Comment on "Increase in voice level and speaker comfort in lecture rooms' [J. Acoust. Soc. Am. 125, 2072-2082 (2009)]," J. Acoust. Soc. Am. 129, 1161-1164 (2011).

${ }^{23}$ D. Pelegrin-Garcia, O. Fuentes-Mendizabal, J. Brunskog, and C.-H. Jeong, "Equal autophonic level curves under different room acoustic conditions," J. Acoust. Soc. Am. 130, 228-238 (2011).

${ }^{24}$ K. Ueno, K. Kato, and K. Kawai, "Effect of room acoustics on musicians' performance. Part I: experimental investigation with a conceptual model," Acta Acust. Acust. 96, 505-515 (2010).

${ }^{25}$ D. Cabrera, D. Lee, R. Collins, B. Hartmann, W. Martens, and H. Sato, "Variation in oral-binaural room impulse responses for horizontal rotations of a head and torso simulator," Build. Acoust. 18, 227-252 (2011).

${ }^{26} \mathrm{~A}$. Gade, "Investigations of musicians' room acoustic conditions in concert halls. Part I: Methods and laboratory experiments," Acustica 69, 193-203 (1989).

${ }^{27} \mathrm{~A}$. Gade, "Investigations of musicians room acoustic conditions in concert halls. Part II: Field experiments and synthesis of results," Acustica 69, 249-262 (1989).

${ }^{28} \mathrm{H}$. Knecht, P. Nelson, G. Whitelaw, and L. Feth, "Background noise levels and reverberation times in unoccupied classrooms: Predictions and measurements," Am. J. Audiol. 11, 65-71 (2002).

${ }^{29}$ E. Pekkarinen and V. Viljanen, "Acoustic conditions for speech communication in class-rooms," Scand. Audiol. 20, 257-263 (1991).

${ }^{30} \mathrm{M}$. Hodgson, "Rating, ranking, and understanding acoustical quality in university class-rooms," J. Acoust. Soc. Am. 112, 568-575 (2002).

${ }^{31}$ ANSI/ASA S12.60-2010: Part 1 American National Standard Acoustical Performance Criteria, Design Requirements, and Guidelines for Schools, Part 1: Permanent Schools (Acoustical Society of America, Melville, NY, 2010).

32“"Acoustic design of schools TSO," Building Bulletin 93, Department of Education and Skills, London (2004).

${ }^{33}$ SS 25268:2007, "Building acoustics—-sound classification of spaces in buildings - health care facilities, educational facilities, day-care centers, offices and hotels," Swedish Standards Institute, Stockholm, Sweden (2007).

${ }^{34}$ Guidelines for Community Noise (World Health Organization Geneva, 1999).

${ }^{35}$ D. Cabrera, H. Sato, W. Martens, and D. Lee, "Binaural measurement and simulation of the room acoustical response from a person's mouth to their ears," Acoust. Aust. 37, 98-103 (2009).

${ }^{36}$ ITU-T P. 58, "Head and torso simulator for telephonometry," Recommendation, International Telecommunication Union, Geneva (1996).

${ }^{37} \mathrm{~W}$. Olsen, "Average speech levels and spectra in various speaking/listening conditions: A summary of the Pearson, Bennett, \& Fidell (1977) report," Am. J. Audiol. 7, 21-25 (1998). 
${ }^{38}$ W. Chu and A. Warnock, "Detailed directivity of sound fields around human talkers," Technical Report, Institute for Research in Construction, National Research Council Canada, Ottawa, Canada (2002).

${ }^{39}$ Brüel \& Kjær, Head and Torso Simulator Type 4128-Product data, Nærum, Denmark (2009).
${ }^{40}$ M. Schroeder, "New method of measuring reverberation time" J. Acoust. Soc. Am. 37, 409-412 (1965).

${ }^{41}$ T. Houtgast and H. Steeneken, "A multi-language evaluation of the RASTI-method for estimating speech-intelligibility in auditoria," Acustica 54, 185-199 (1984). 